\title{
EVALUACIÓN DE LA SOBRECARGA DE CUIDADORAS/ES INFORMALES
}

\section{EVALUATION OF BURDEN IN INFORMAL CAREGIVERS}

\author{
ViVIANE Jofré AravenA* \\ Olivia Sanhueza Alvarado**
}

\begin{abstract}
RESUMEN
Objetivo: Establecer la sobrecarga derivada de la tarea de cuidar de las/los cuidadoras/es informales y los factores asociados a ella. Material y método: Diseño descriptivo, correlacional en una muestra accidental por conglomerado de cuidadoras/es informales de pacientes adscritos al programa de postrados permanentes de los centros de salud pertenecientes a la DAS de Concepción, Chile. Se registraron las variables sociodemográficas relacionadas con la actividad de cuidar, además de la sobrecarga del cuidador, con la escala de Sobrecarga de Zarit. Se exploraron las dimensiones latentes de la escala de sobrecarga para determinar la multidimensionalidad de este fenómeno. Los datos se registraron en una base de datos ingresados en el programa estadístico SPSS versión 15.0, se les aplicó estadística descriptiva e inferencial. Resultados: Los resultados son compatibles con la mayoría de los estudios respecto de las características de las/los cuidadoras/es informales. El perfil es una mujer entre 54 y 65 años de edad, hija, esposa y/o madre, que cuida más de 8 horas diarias a su familiar y realiza esta tarea desde hace más de 6 años, que no ha recibido la formación para los diferentes cuidados que otorga y que presenta niveles moderados de sobrecarga. Conclusiones: Es posible establecer algunos parámetros predictivos de sobrecarga, cuidadoras/es que muestran escasas redes sociales, estado de salud regular y peor que hace un año atrás y que presentan molestias físicas. Es prioridad que en nuestro sistema sanitario el/la cuidador/a informal sea tomada en cuenta como un usuario que también requiere de atención y no sólo visto/a como un/a agente colaborador/a para el sistema formal de salud.
\end{abstract}

Palabras clave: Cuidadora/or informal, carga del cuidador, enfermedad crónica.

\begin{abstract}
Objective: To determine the amount of caregiver burden and related factors on informal caregivers. Methods: A descriptive, correlational study was conducted using a convenience sample of informal caregivers of patients assigned to the prostrate health program in the Municipality of Concepción, Chile. Sociodemographic variables were recorded and related to the activity of caregiving, as well as caregiver stress, using the Zarit Burden Scale. Latent dimensions were explored using the Burden Scale to determine the multidimensionality of the phenomenon. Data were recorded in a database and entered into SPSS Statistical Program Version 15.0 and descriptive and inferential statistics were applied. Results: The results are consistent with most studies on the characteristics of the informal caregiver. The profile is a female between ages 54 and 65 who is a daughter, wife and/or mother who cares for her sick family member for more than 8 hours daily, has carried out this work for over 6 years, has not received caregiver training and presents with moderate levels of stress. Conclusions: It is possible to predict caregiver burden in cases where social networks are poor, where health status in the caregiver is worse than one year earlier, and where the caregiver has some physical ailments. It should be a priority in our health system that informal caregiver burden be given attention and that the informal caregiver role not seen simply as adjunctive or collaborative to the formal health system.
\end{abstract}

Key words: Informal caregiver, informal caregiver burden, chronic disease.

Fecha recepción: 14/09/2009 Fecha aceptación: 16/12/2010

\footnotetext{
*Enfermera. Docente Depto. Enfermería. Universidad de Concepción. Concepción, Chile. E-mail: vjofre @udec.cl

${ }^{* *}$ Enfermera. Docente Depto. Enfermería. Universidad de Concepción. Concepción, Chile. E-mail: osanhue@udec.cl
} 


\section{INTRODUCCIÓN}

El cambio que se ha producido en los últimos años en la pirámide poblacional de los países occidentales ha dado lugar a variados fenómenos, como es el aumento de la población en edad geriátrica y una mayor supervivencia de personas con enfermedades crónicas y discapacidades físicas y/o psíquicas, lo cual también implica un aumento en la necesidad de personas que se dediquen a brindar cuidados en el ambiente familiar.

Aunque toda la familia se ve afectada, en la mayoría de los casos es sólo un individuo el que asume el cuidado. Esta persona generalmente es un integrante de la familia, quien toma el rol de cuidador/a y quien, a su vez, requiere adaptarse a las demandas del cuidado (1).

Tradicionalmente, son las mujeres en quienes recae la atención del cuidado de los miembros de la familia, no importando la edad de la persona que demanda atención. Parte del rol femenino ha sido asumir responsabilidad por el cuidado de los enfermos de la familia (2) al interior del hogar, lo que conlleva dificultades frente a la posibilidad de esta población de insertarse de modo igualitario en el ámbito laboral, social y económico de la sociedad.

Gran parte de la investigación sobre cuidados informales se ha centrado en el análisis de las consecuencias que tiene sobre la cuidadora/or el hecho de atender la salud de sus familiares y el impacto y la angustia correspondiente que le produce asumir los cuidados de un familiar. Este proceso de percepción del impacto de los cuidados y la angustia (distress) que se le asocia es lo que se denomina sobrecarga de la cuidadora/or (3).

La sobrecarga hace referencia a la valoración que hace la/el cuidadora/or de los estresores que se asocian con el cuidar y su impacto $(4,5,6)$, al conjunto de estresores primarios y secundarios asociados a los cui- dados (7), al grado de implicación de los cuidadores en los cuidados, a las consecuencias que se derivan de cuidar sobre sus vidas (8, 9).

Zarit (8), Miller y Montgomery (10) diferencian entre sobrecarga personal, referida a la valoración que hace la cuidadora del grado en que sus actividades personales se encuentran limitadas, y sobrecarga interpersonal, que refleja la percepción que tiene la cuidadora de las conductas problemáticas de la persona que cuida.

Ambos autores han diferenciado entre sobrecarga objetiva, conceptualizada como el grado de dedicación a los cuidados y como impacto de los cuidados en la vida de la cuidadora; y sobrecarga subjetiva, que se refiere a la forma en la que la cuidadora responde a la situación de cuidados, se define como el sentimiento psicológico que se asocia al hecho de cuidar y se relaciona con el grado en que la cuidadora se siente agotada y percibe dificultades para manejar la situación de cuidados.

En un estudio realizado en Chile, con cuidadores de pacientes con SIDA, se concluyó que los cuidadores eran mujeres, en su mayoría madres, que vivían con su hijo(a) enfermo(a) y que llevaban por lo menos dos años ejerciendo el rol de cuidadora, las cuales percibían como carga el desgaste emocional y el económico, con alta posibilidad de estrés emocional debido al largo tiempo que llevaban cumpliendo su rol y viviendo a diario con la persona enferma (11).

En otro estudio, donde la muestra estuvo constituida por cuidadoras informales de pacientes crónicos, se encontró que el 44\% de la muestra presentaba enfermedades crónicas (hipertensión, diabetes, asma, várices, depresión y otras), entre los síntomas percibidos se destacan cefaleas, cansancio, dorsalgias, sensación de depresión, insomnio, dolores articulares y fatigas. Se identificaron problemas psicoafectivos, donde el mayor porcentaje se derivaba del rol cuidador (34\%) y el $12 \%$ se originaba a partir 
del sentimiento de culpabilidad por delegar funciones del cuidado. Todas las mujeres describieron pérdida de amistades, cambios de hábitos de vida, sentimientos negativos por la persona que cuidan, conflictos de rol hacia la familia, abandono de las actividades laborales, deterioro en las comunicaciones conyugales y descuido en la imagen corporal. Respecto al estado de salud, el $57 \%$ percibía su salud física como regular; el 65\% describía su salud emocional como regular; el 39\% percibía su estado de salud cognitivo como regular y el $63 \%$ percibía su estado de salud social como bueno (12).

En un trabajo del tipo bibliográfico, sobre la carga de cuidadores familiares de personas con Accidente Cerebro Vascular $(\mathrm{ACV})$, realizado en Brasil, se encontró que los aspectos generadores de carga se resumen en las categorías: aislamiento social, insatisfacciones conyugales, dificultades financieras y déficit en la salud física y en el autocuidado (13).

En una investigación realizada en la Habana, Cuba, se encontró que los principales síntomas que presentaban los cuidadores de ancianos se relacionaban con el sistema osteomioarticular, con más del $60 \%$ aproximadamente de incidencia, seguido de los síntomas generales. El más frecuente correspondió a las artralgias. Respecto a los síntomas psíquicos del "cuidador", hubo predominio de la depresión, cansancio y trastornos del sueño. En los problemas sociales, se destacó la disminución o pérdida del tiempo libre, la soledad y el aislamiento fueron los aspectos que más repercutieron en la muestra estudiada (14).

Escudero, Díaz, Pascual (15) realizaron un estudio observacional, descriptivo y transversal con 32 cuidadores mediante una entrevista presencial domiciliaria. Los resultados del estudio ratifican que los cuidadores informales son fundamentalmente mujeres. Se observa un grupo de cuidadores más jóvenes (hijos), con un ligero incremento de hombres. Los autores plantean que cuidar a familiares dependientes o con enfermedades crónicas supone estados de nerviosismo, falta de descanso, problemas para dormir, dificultad para relacionarse socialmente, dolores físicos y necesidad de vacaciones. Establecen que las familias son solidarias con los dependientes y los mantienen en el hogar, señalando que las principales razones para cuidar de sus familiares son el cariño y el deber. A pesar de que los cuidadores mayoritariamente cuentan con ayudas informales que provienen de su red de relaciones familiares y sociales, existe un porcentaje que manifiesta no disponer de las mismas. Con relación a las ayudas que les gustaría recibir del sistema formal, aceptan lo que reciben sin cuestionamientos. Es importante destacar que los cuidadores perciben que las ayudas se dirigen fundamentalmente a los dependientes $y$ no a ellos.

En un estudio realizado con 90 cuidadores de pacientes con esquizofrenia, en el cual el objetivo era evaluar su sobrecarga, se encontró que la edad, el sexo, la clase social, el tener pareja o las horas de dedicación al cuidado del paciente no influyeron en los niveles de sobrecarga medidos(16).

En otro estudio, en el que se midió la sobrecarga con la escala de Zarit para estimar la probabilidad de desarrollar un trastorno psiquiátrico a 40 cuidadores de pacientes con Alzheimer, en la que en su mayoría correspondían a hijas de los enfermos, se encontró que un puntaje de 63 o más de sobrecarga es un predictor de distrés psicológico en los cuidadores (17).

El objetivo de este estudio fue establecer cuál es el nivel de sobrecarga de los cuidadores y las variables que se relacionan con ésta. Es un intento de contribuir a visibilizar la realidad a la cual se enfrentan muchos hogares y visibilizar esta práctica de manera de considerar a las cuidadoras/es como un usuario más de las intervenciones y prestaciones que el sistema de salud formal debe otorgar. 


\section{MATERIAL Y MÉTODO}

Diseño descriptivo, correlacional en una muestra accidental por conglomerado de cuidadoras/es informales de pacientes adscritos al programa de postrados permanentes de los centros de salud pertenecientes a la DAS de Concepción, Chile, con un error muestral de $6,5 \%$ y con un $95 \%$ de confianza, respetando los porcentajes de cada centro de salud, equivalente a 156 casos.

Para la obtención de los datos se diseñó un instrumento que indagó variables sociodemográficas y antecedentes relacionados con la actividad de cuidar. Para evaluar la carga subjetiva asociada al cuidado, se utilizó el Caregiver Burden Interview de Zarit validada por Martín et al. (18), éste consiste en una escala tipo likert de 22 ítemes, que mide el grado en que los cuidadores perciben cómo sus responsabilidades tienen efectos adversos sobre su salud, vida personal y social, finanzas y bienestar emocional. Cada ítem se puntúa según la severidad de la repercusión del 0 al 4 . El puntaje varía de 0 a 88 puntos, donde el mayor valor informa mayores niveles de sobrecarga. Este instrumento se ha mostrado válido y confiable (19).

Se realizó una prueba piloto en un grupo de 22 cuidadoras informales de pacientes pertenecientes al programa de postrados de un centro de salud de otra comuna, para comprobar la validez y confiabilidad de la Escala de Sobrecarga del cuidador de Zarit. La aplicación de la prueba piloto a este grupo permitió evaluar que no existen dificultades para la comprensión y entendimiento del instrumento. Se realizó el análisis de fiabilidad obteniéndose un Alfa de Cronbach de 0.907 , lo que muestra una alta consistencia.

Previo a la aplicación del instrumento, el protocolo de investigación fue aprobado por el comité de ética de la Facultad de Medici- na de la Universidad de Concepción, y las unidades de análisis aceptaron participar del estudio a través de la firma de un consentimiento informado.

Para la organización, presentación y análisis exploratorio y relacional de los datos se utilizó el programa estadístico SPSS versión 15.0, utilizándose estadística descriptiva para determinar el perfil de la muestra. Para determinar la multidimensionalidad de la sobrecarga se utilizó análisis factorial con rotación varimax para la escala Zarit, extrayéndose 4 factores que explican el $64.9 \%$ de la varianza.

Con el fin de aumentar la validez de constructo del instrumento y mejorar los valores de confiabilidad, fue necesario la eliminación de algunos ítemes de la escala, pues mostraron un comportamiento poco claro en la determinación de la sobrecarga del/la cuidador/a.

Con los ítemes que conformaron cada factor fue posible construir 4 dimensiones que reflejan cómo la tarea de cuidar a otros afecta distintos aspectos de la vida de la/el cuidadora/or.

El primer factor dice relación con el grado que la relación con su familiar le afecta, sintiéndose tensa/o, agotada/o, sin vida privada, sin tiempo y puede ser denominado Impacto del cuidado. El segundo factor está representado por el grado de interferencia en las relaciones interpersonales, el cual puede denominarse Relaciones Interpersonales. El tercer factor, denominado Expectativas de Autoeficacia, refleja sentimientos de incapacidad frente a las demandas con su familiar. El cuarto factor que resultó al realizar la reducción de complejidad, representado sólo por un ítem, está relacionado con la sensación que es el/la único/a que puede cuidar a su familiar, el cual puede denominarse Dependencia.

El espacio factorial se construyó como se muestra en la Tabla 1. 
Tabla 1. Estructura factorial, varianza porcentual y confiabilidad de la Escala Zarit.

\begin{tabular}{|l|l|c|c|c|}
\hline \multicolumn{1}{|c|}{ Factor } & \multicolumn{1}{|c|}{ Ítemes } & \% varianza & $\begin{array}{c}\text { \%varianza } \\
\text { acumulada }\end{array}$ & $\begin{array}{c}\text { Alfa de } \\
\text { Cronbach }\end{array}$ \\
\hline 1. Impacto del Cuidador & $1,2,3,8,10$ & 34.07 & 34.07 & $\mathbf{0 . 7 6 5}$ \\
\hline 2. Relaciones Interpersonales & $5,9,11,12,16$ & 13.25 & 47.32 & $\mathbf{0 . 7 7 9}$ \\
\hline 3. Expectativas de autoeficacia & 19,20 & 9.33 & 56.65 & $\mathbf{0 . 7 9 8}$ \\
\hline 4. Dependencia & $\mathbf{1 3}$ & $\mathbf{8 . 2 4}$ & $\mathbf{6 4 . 8 9}$ & - \\
\hline
\end{tabular}

Los valores de fiabilidad de los componentes extraídos de la escala de Zarit fueron aceptables, al igual que el valor para la escala total que arrojó un Alfa de Cronbach de 0.822 .

Para contrastar las variables se aplicó un análisis de varianza no paramétrico debido a la distribución no normal de los componentes de la Escala Zarit.

Para las variables dicotómicas se utilizó la prueba de U Mann-Whitney que permite comparar las medias de dos grupos independientes. Para las variables con más de tres categorías se usó la prueba de KruskalWallis que es el equivalente no paramétrico del Anova.

\section{RESULTADOS}

La muestra de este estudio se conformó por un $88.5 \%$ de mujeres, $64.7 \%$ vive en pareja y la mayoría (92.9\%) practica alguna religión, siendo el $64.7 \%$ católica.

El mayor porcentaje posee un nivel de educación media (42.9\%), el 63.5\% no recibe remuneración por la actividad que realiza y el mayor porcentaje (62.2\%) no ejerce como jefe de hogar. Respecto de la edad de este grupo de cuidadoras/es, el mayor porcentaje se encuentra en el rango de entre 54 y 65 años.

La mayoría (85.3\%) de las/os cuidadoras/ es de este estudio cuida a una sola persona con la que posee algún parentesco (86.6\%), de ellos el 74,4\% corresponde fundamentalmente a hijas/os, esposas y hermanas el 12.2 $\%$ son otros parientes por afinidad como sobrinas y nueras; todas dedican más de 8 horas al cuidado durante los días de semana $(87.8 \%)$ y durante el fin de semana continúan haciéndolo, aumentando la cantidad de cuidadoras/es que dedica más de 8 horas al cuidado, a un $90.4 \%$. Respecto al tiempo que lleva ejerciendo esta tarea, el 33.3\% lo realiza desde hace un mes hasta 3 años, 46.8\% de la muestra lo hace desde hace 10 años y más. Sólo el 32.7\% de estas/os cuidadoras/es ha recibido formación para brindar cuidados, fundamentalmente desde los centros de salud. Los cuidados que brindan diariamente estas personas van desde los propios de la vida diaria (36.5\%): como aseo, alimentación, ayudar a vestirse; incluyendo cuidados específicos de menor complejidad (27.5\%): como es administración de medicamentos, ejercicios específicos, control de dieta, control de signos vitales, etc., hasta cuidados de mayor complejidad en un 35.9\%, que corresponden a sondeo, inyecciones intramusculares y subcutáneas, curaciones, cambio de bolsas de colostomía, alimentación por sonda, oxigenoterapia y manejo de algunos monitores.

La mayor parte $(87.2 \%)$ de las/os cuidadores de este estudio refieren presentar molestias y/o dolores en las últimas dos semanas, más de la mitad 52,6\% considera su estado de salud como regular, y al compa- 
rarlo con un año atrás, el 50.6\% refiere que se encuentra igual, el 36.5\% lo califica como peor y sólo el $12.8 \%$ encuentra que su estado de salud es mejor.

La distribución de las molestias presentadas por la muestra es en primer lugar el dolor de piernas en un $60.3 \%$, el 55.2\% presenta dolor de espalda, cansancio lo manifiesta el $45.5 \%$ y dolor de cabeza $43.6 \%$. Siendo siempre mayores los porcentajes de molestias presentadas por las mujeres versus los hombres.

Respecto a la carga del cuidador las/os cuidadoras/es obtuvieron puntajes desde 5 a 76 puntos (de 88 máximo), lo cual indica valores moderados de sobrecarga para esta muestra de cuidadoras/es informales, con una media de 33.141 y una desviación de 15.10 puntos.

Al aplicar la prueba de U Mann-Whitney se observaron valores de rangos promedios superiores para la carga del cuidador en las mujeres en el componente de Impacto del cuidador y para los hombres, valores de rangos promedios más altos en el componente de Dependencia. No existiendo diferencias significativas respecto al sexo, aunque en el Impacto del cuidador la significancia es cercana (0.086), lo cual muestra una tendencia mayor en las mujeres de sobrecarga derivadas de la tarea de cuidar a otros.

Respecto a la situación de pareja, se apreciaron mayores rangos promedios de carga en las/os cuidadoras/es sin pareja en los componentes Impacto del cuidador y Expectativas de autoeficacia, sin embargo estas diferencias no son estadísticamente significativas.

En relación a la actividad, categorizada en remunerada, no remunerada y otras que incluye a aquellos/as cuidadoras/es que se encuentran jubiladas/os, éstos mostraron menores rangos promedios de sobrecarga en las Expectativas de autoeficacia, respecto a las otras dos categorías, sin relación estadísticamente significativa en el valor de Chicuadrado.

En el nivel educacional se observaron ma- yores rangos promedios de sobrecarga en los componentes de: relaciones interpersonales, expectativas de autoeficacia y dependencia en las/os cuidadoras/es sin educación, respecto a las otras categorías (educación bási$\mathrm{ca}$, media y superior).

Las/os cuidadoras/es que manifestaron no pertenecer a ninguna religión presentaron rangos promedios de mayor carga en los componentes de Impacto del cuidador, relaciones interpersonales y expectativas de autoeficacia, sin observarse diferencias significativas.

Respecto a la percepción con el ingreso económico familiar, mostraron mayores rangos promedios de sobrecarga en los cuatro componentes las/os cuidadoras/es que perciben que el ingreso económico cubre muy bien sus necesidades básicas, aunque no hubo diferencias estadísticamente significativas con los otros grupos.

En la variable jefatura del hogar, se encontraron rangos promedios mayores de carga en los componentes de impacto de cuidar y expectativas de autoeficacia en aquellas/os cuidadoras/es que no ejercen el rol de jefes de hogar, en cambio los/as cuidadores/as que sí ejercen jefatura de hogar presentan rangos promedios mayores de carga en el componente de Dependencia.

Respecto al número de personas que cuida, aquellas/os cuidadoras/es que cuidan a más de un familiar, habitualmente ambos padres, presentaron rangos promedios más altos de sobrecarga, sin haber diferencias significativas entre los grupos.

En la variable horas del día que dedica al cuidado, durante los días de la semana y durante los fines de semana, categorizada como habitual, cuando dedica hasta 8 horas del día y no habitual cuando el tiempo es mayor, se evidenció que las/los cuidadoras/es que cuidan en forma habitual los días de semana y fines de semana, tienen valores promedios superiores de sobrecarga en los componentes de relaciones interpersonales y en el de expectativas de autoeficacia. 
Para la variable tiempo que ejerce la labor de cuidador/ra, en general se observan rangos promedios elevados en todos los componentes para todos los grupos, no habiendo relación con los diferentes tiempos que ejercen la labor de cuidadoras/es. En el primer componente aquellas cuidadoras/es que ejercen su labor de 5 a 10 años tienen el más alto rango, al igual que en el componente de Relaciones interpersonales. En las expectativas de autoeficacia el grupo que ejerce la labor entre 3 a 5 años presentó el rango promedio más elevado. Y aquellas cuidadoras/es que lo hacen desde más de 10 años tienen el rango más alto en el componente de dependencia. No existiendo diferencias estadísticamente significativas en los diferentes grupos.

En todos los componentes se observó rangos promedios de sobrecarga más altos para el grupo que no recibió instrucción para realizar la tarea de cuidar. Aun cuando no se observan diferencias significativas en ninguno de los grupos.

Al contrastar la variable Tipo de Cuidados que brinda, a través de la prueba de KruskalWallis, se observaron rangos promedios de sobrecarga más elevados en los componentes de Impacto del cuidador y Dependencia en las/os cuidadoras/es que brindaban cuidados de mayor.

El grupo que brinda cuidados específicos de menor complejidad obtuvo rangos promedios más altos de sobrecarga en el componente de Relaciones interpersonales sin evidenciar una diferencia significativa (valor $\mathrm{p}<0.288)$ en los grupos.

El grupo que brinda cuidados propios de la vida diaria obtuvo rangos promedios superiores de sobrecarga en el componente expectativas de autoeficacia, sin una diferencia significativa (valor $-\mathrm{p}<0.079$ ) entre las distintas categorías.

Respecto a la presencia de molestias en las últimas dos semanas, aquellas/os cuidadoras/es que sí presentaron molestias, mostraron rangos promedios superiores de carga para los componentes Impacto del cuidador,
Relaciones interpersonales y Dependencia, encontrándose diferencias significativas (valor $\mathrm{p}<0.027$ ) en el primer componente con aquellas/os cuidadoras/es que no presentaban molestias en las últimas dos semanas.

Para la variable Estado de Salud se obtuvieron rangos promedios más altos para el grupo de cuidadoras/es que perciben su salud como regular en todos los componentes de la escala de carga del cuidador, con excepción del componente expectativas de autoeficacia en que levemente presentó valores de rangos promedios más altos el grupo que percibe su salud como buena. Los valores de Chi cuadrado muestran diferencias significativas para los componentes de impacto del cuidador $(\mathrm{p}<0.30)$ y dependencia $(\mathrm{p}<0.30)$.

Respecto a la percepción del Estado de salud comparado con un año atrás se encontraron rangos promedios más altos para el grupo que percibe su estado de salud peor que hace un año en todos los componentes de la escala de carga del cuidador, con excepción del componente expectativas de autoeficacia, con diferencias significativas en los componentes Impacto del cuidador $(\mathrm{p}<0.027)$ y relaciones interpersonales $(\mathrm{p}<0.013)$.

\section{DISCUSIÓN}

Sin duda, la caracterización de esta muestra de cuidadoras/es de los pacientes pertenecientes al programa de Postrados de la DAS de Concepción, es coincidente con los escasos estudios nacionales $(11,12,20)$ y con los estudios internacionales, como los efectuados por García-Calvente et al. $(3,7,14,16)$. Quien realiza la labor de cuidar a sus familiares en el hogar es una mujer, generalmente hija, esposa y/o hermana en edades en las cuales ellas mismas deberían ser objeto de acciones preventivas para evitar el desarrollo de enfermedades crónicas, físicas o psicológicas, derivadas de la sobrecarga que significa estar presente para otro(21), considerando 
además que un alto porcentaje lleva ejerciendo está tarea desde hace 10 años y más, sin haber recibido en muchas oportunidades la formación para brindar estos cuidados, que les ocupan una gran cantidad de horas del día, de lunes a domingo, en forma paralela a sus otras actividades de dueña de casa, ya que además la mayoría vive en pareja, esto probablemente se explica por los roles que tradicionalmente han sido asignados a las mujeres, cultural y socialmente $(3,22,23)$.

A pesar de los niveles moderados de sobrecarga, considerándola como un fenómeno unidimensional, que evidenciaron estas/ os cuidadoras/es, es preciso considerar la multidimensionalidad de este fenómeno y valorar las variables que se relacionan con mayores rangos promedios en las dimensiones identificadas

En la dimensión Impacto del Cuidador se observan mayores rangos promedios de sobrecarga en: mujeres, sin pareja, sin religión, no jefas de hogar, que brindan cuidados de mayor complejidad, que presentan molestias en las últimas dos semanas y que perciben su salud como regular, peor que hace un año.

En la dimensión de Relaciones Interpersonales, presentaron mayores rangos promedios de sobrecarga aquellas/os cuidadoras/ es sin educación, sin religión, que brindan cuidados de menor complejidad y que perciben su salud como regular, peor que hace un año.

Para la dimensión Expectativas de autoeficacia, los mayores rangos promedios de sobrecarga se observaron en las/os cuidadoras/es sin pareja, sin educación, sin religión, no jefas de hogar, y que brindan cuidados propios de la vida diaria.

En la dimensión Dependencia, los hombres, sin educación, que son los jefes de hogar, que brindan cuidados de mayor complejidad, que presentaron molestias en las últimas dos semanas y que perciben su salud como regular, presentaron mayores rangos de sobrecarga.

Es posible plantear que a pesar de los po- cos resultados con significancia estadística al contrastar las variables independientes con las 4 dimensiones latentes de la escala de Zarit, los hallazgos permiten establecer con seguridad algunos parámetros predictivos de sobrecarga, relacionadas con el riesgo de desarrollar alteraciones psicológicas, tal como lo plantea el estudio español realizado por Martin Carrasco et al. (17).

Aquellas/os cuidadoras/es que muestran escasas redes sociales, mostrarían mayor riesgo de sobrecarga (sin pareja, sin religión, sin educación)(24).

El estado de salud y la presencia de molestias serían variables a considerar siempre al momento de evaluar las características de las personas que cuidan a sus familiares en casa, y de esta forma abordar las necesidades de cuidado de los cuidadores.

\section{CONCLUSIONES}

Los resultados presentados proveen una base para futuras investigaciones, que permitan explorar otras características y su relación con la sobrecarga de la tarea de cuidar.

Es preciso evidenciar que el ser cuidador no esta definido por el enfermo a quien cuida, sino que por la actividad que supone ser cuidador.

Es prioridad que en nuestro sistema sanitario el cuidador/ra informal sea tomada en cuenta como un usuario que también requiere de atención y no sólo ser visto/a como un agente colaborador para el sistema formal de salud.

Se hace necesario que el Sistema de Salud proponga la creación de instancias que permitan a estas personas, que contribuyen silenciosamente con la salud de la comunidad, el desahogo de sus actividades, con la instauración de hospitales de día, o instituciones de cuidados de larga duración, que serán cada vez más necesarias dada la mayor esperanza de vida, con el consiguiente aumento 
de personas que no sólo padecen enfermedades crónicas sino que también personas más dependientes debido a la pérdida de habilidades no sólo motoras, sino que también intelectuales y que requerirán mayor cuidado y supervisión.

En todo este cambio, la disciplina de enfermería deberá asumir un papel protagónico, en el sentido de adelantarse a los cambios, formulando a corto plazo currículos con énfasis en lo social y comunitario para poder planificar estrategias que permitan efectivamente brindar apoyos concretos no sólo a los enfermos, sino que además a las personas que por múltiples razones asumen la responsabilidad de cuidar a otros, sin tener que verse menoscabadas en su desarrollo personal y mucho menos deteriorando su estado de salud.

\section{REFERENCIAS}

1. Wykle M, Cellar F. Investigación en entrega de cuidados a las familias y enfermedades crónicas. Documento. Universidad de Case Western Reserve Cleveland, Ohio. 2000.

2. Sánchez-Ayéndez M. Mujeres puertorriqueñas de edad mediana como principales proveedoras de cuidado a adultos/as mayores. Cuadernos Mujer salud 1999; 4:124-135.

3. García - Calvente MM, Mateo Rodríguez I, Gutiérrez Cuadra P. Cuidados y cuidadores en el sistema informal de salud: investigación cuantitativa. Granada: Escuela Andaluza de Salud Pública. 1999.

4. Lawton Mp, Keblan MH, Moss M, Rovine M, Glicksman A. Measuring care giving appraisal. Journal of Gerontology: Psychological Sciences 1989; 44:61-71.

5. Miller B, Mc Fall S. The effect of caregiver's burden on change in frail older person's use of formal helpers. J Health Social Behav.1991a; 32:165-79.
6. Miller B, Mc Fall S. Stability and change in the informal task suppot network of frail older persons. Gerontologist 1991b;31(6): 735-45.

7. Pearlin LI. The careers of caregivers. Gerontologist 1991;32(5): 647.

8. Zarit SH, Toseland RW. Current and future direction in family caregiving research. Gerontologist 1989;29:481-3.

9. Zarit SH. Behavioral disturbances of dementes and caregiver issues. Int Psychogeriatr 1996;8 (Suppl. 3):263-8.

10. Miller B, Montgomery A. Family caregivers and limitations in social activities. Res Aging 1990;12(1): 72-93.

11. Cazenave A, Ferrer X, Castro S, Cuevas S. El familiar cuidador de pacientes con SIDA y la toma de decisiones en salud. Rev Chil Infect 2005; 22(1): 51-57.

12. Jofré VA, Mendoza SP. Toma de decisiones en salud en mujeres cuidadoras informales. Cienc Enferm 2005; 11(11): 37-49.

13. Bocchi S. Viviendo la sobrecarga al convertirse en cuidador familiar de personas con accidente cerebrovascular: análisis del conocimiento. Rev Latino-Am Enfermagem. 2004; 12(1): 115-121.

14. Lara Pérez L, Díaz Díaz M, Herrera Cabrera E, Silveira Hernández P. Síndrome del cuidador en una población atendida por equipo multidisciplinario de atención geriátrica. Revista Cubana de Enfermería 2001; 17(2): s/p.

15. Escudero B, Díaz E, Pascual O. Cuidadores informales. Necesidades y ayudas. Rev Rol Enf 2001; 24(3): 183-189.

16. Leal MI, R. Sales R, Ibáñez E, Giner J. Valoración de la sobrecarga en cuidadores informales de pacientes con esquizofrenia antes y después de un programa psicoeducativo. Actas Esp Psiquiatr 2008; 36(2): 63-69.

17. Martin Carrasco M, et al. Sobrecarga de pacientes con enfermedad de Alzheimer y distrés psíquico, Una asociación relegada en la valoración de las demencias. Ac- 
tas Esp Psiquiatr. 2002; 30(4): 201-206.

18. Ginsberg J, Martínez MF, Mendoza A, Pabón JL. Carga subjetiva percibida por el cuidador y su relación con el nivel de deterioro de pacientes con diagnóstico de demencia. Archivos Venezolanos de Psiqiatría y Neurología 2005; 51(104): 7-11.

19. Badia X, Salamero M, Alonso. La medida de la salud. Guía de Escalas de Medición en español. Fundación Lilly. 2002.

20. Vidal D, Zavala M, Castro M, Quiroga P, Klassen G. El significado del paciente con demencia para el cuidador, en una comunidad urbana y rural. Revista de Servicio Social. 1999; 1(2): s/p.

21. Espín A. Caracterización psicosocial de cuidadores informales de adultos mayores con demencia. Rev. cub. salud pública 2008; 34(3): 1-12.

22. Murillo de la Vega S. La invisibilización del cuidado en la familia y los sistemas sanitarios. Política y Sociedad. Universidad de Salamanca 2000; 35: 73-80.

23. García-Calvente MM, Del Río Lozano M, Eguiguren AP. Desigualdades de género en el cuidado informal a la salud. Revista Vasca de sociología y ciencia política. 2007; (44): 291-308.

24. Montalvo A, Flores I. Características de los cuidadores de personas en situación de cronicidad. Cartagena (Colombia) Un estudio comparativo. Salud Uninorte. Barranquilla 2008; 24(2): 181-190. 